\title{
Genome-wide association study followed by trans-ancestry meta-analysis identify 17 new risk loci for schizophrenia
}

Jiewei Liu', Shiwu Li $i^{1,2}$, Xiaoyan Li ${ }^{1,2}$, Wenqiang Li, ${ }^{3,4}$, Yongfeng Yang ${ }^{3,4}$, Suqin Guo ${ }^{3,4}$, Luxian Lv ${ }^{3,4}$, Xiao Xiao , Yong-Gang Yao ${ }^{1,2,5,6}$, Fanglin Guan ${ }^{7}$, Ming Li $\mathrm{Li}^{1,2,5,6}$ and Xiong-Jian Luo ${ }^{1,2,5,8^{*}}$

\begin{abstract}
Background: Over 200 schizophrenia risk loci have been identified by genome-wide association studies (GWASs). However, the majority of risk loci were identified in populations of European ancestry (EUR), potentially missing important biological insights. It is important to perform 5 GWASs in non-European populations.

Methods: To identify novel schizophrenia risk loci, we conducted a GWAS in Han Chinese population (3493 cases and 4709 controls). We then performed a large-scale meta-analysis (a total of 143,438 subjects) through combining our results with previous GWASs conducted in EAS and EUR. In addition, we also carried out comprehensive post-GWAS analysis, including heritability partitioning, enrichment of schizophrenia associations in tissues and cell types, trancscriptome-wide association study (TWAS), expression quantitative trait loci (eQTL) and differential expression analysis.

Results: We identified two new schizophrenia risk loci, including associations in SHISA9 (rs7192086, $P=4.92 \times 10^{-08}$ ) and PES1 (rs57016637, $P=2.33 \times 10^{-11}$ ) in Han Chinese population. A fixed-effect meta-analysis (a total of 143,438 subjects) with summary statistics from EAS and EUR identifies 15 novel genome-wide significant risk loci. Heritability partitioning with linkage disequilibrium score regression (LDSC) reveals a significant enrichment of schizophrenia heritability in conserved genomic regions, promoters, and enhancers. Tissue and cell-type enrichment analyses show that schizophrenia associations are significantly enriched in human brain tissues and several types of neurons, including cerebellum neurons, telencephalon inhibitory, and excitatory neurons. Polygenic risk score profiling reveals that GWAS summary statistics from trans-ancestry meta-analysis (EAS + EUR) improves prediction performance in predicting the case/control status of our sample. Finally, transcriptome-wide association study (TWAS) identifies risk genes whose cis-regulated expression change may have a role in schizophrenia.

Conclusions: Our study identifies 17 novel schizophrenia risk loci and highlights the importance and necessity of conducting genetic study in different populations. These findings not only provide new insights into genetic etiology of schizophrenia, but also facilitate to delineate the pathophysiology of schizophrenia and develop new therapeutic targets.
\end{abstract}

Keywords: Schizophrenia, GWAS, Han Chinese, PRS, TWAS, Meta-analysis

\footnotetext{
* Correspondence: luoxiongjian@mail.kiz.ac.cn

${ }^{1}$ Key Laboratory of Animal Models and Human Disease Mechanisms of the

Chinese Academy of Sciences \& Yunnan Province, Kunming Institute of

Zoology, Chinese Academy of Sciences, Kunming 650223, Yunnan, China

${ }^{2}$ Kunming College of Life Science, University of Chinese Academy of

Sciences, Kunming 650204, Yunnan, China

Full list of author information is available at the end of the article
}

C C The Author(s). 2021 Open Access This article is licensed under a Creative Commons Attribution 4.0 International License, which permits use, sharing, adaptation, distribution and reproduction in any medium or format, as long as you give appropriate credit to the original author(s) and the source, provide a link to the Creative Commons licence, and indicate if changes were made. The images or other third party material in this article are included in the article's Creative Commons. licence, unless indicated otherwise in a credit line to the material. If material is not included in the article's Creative Commons licence and your intended use is not permitted by statutory regulation or exceeds the permitted use, you will need to obtain permission directly from the copyright holder. To view a copy of this licence, visit http://creativecommons.org/licenses/by/4.0/. The Creative Commons Public Domain Dedication waiver (http://creativecommons.org/publicdomain/zero/1.0/) applies to the data made available in this article, unless otherwise stated in a credit line to the data. 


\section{Background}

Schizophrenia (SCZ) is a devastating mental disorder that affects about $0.5-1 \%$ of the world's population [1]. The main symptoms of SCZ include positive symptoms (hallucinations and delusions), negative symptoms (anhedonia, alogia, and avolition), and cognitive impairments (impaired working memory and executive function) [2]. Due to the high mortality and considerable morbidity [3], SCZ imposes substantial economic burden on society and becomes a major threat to global health [4]. The pathophysiology of SCZ remains largely unknown. Nevertheless, lines of evidence indicate that SCZ has a strong genetic component. The heritability of SCZ was estimated around 0.80 [5], implying the major role of the inherited variants in SCZ. To dissect the genetic basis of SCZ, great efforts have been made and significant progresses have been achieved. Low-frequency variants such as structural variants [6], copy number variations [7-10], rare [11], and de novo mutations [1216] were reported to be associated with SCZ. In addition, GWASs have identified over 200 risk loci that showed robust associations with SCZ [17-27].

Although recent large-scale studies have provided important insights into the genetic etiology of SCZ, challenges remain in dissecting the genetic architecture of SCZ. First, the majority of risk loci were identified in populations of European ancestry [21, 26]. Considering the diverse differences of allelic frequency and linkage disequilibrium pattern in different continental populations [28], performing GWAS in non-European populations will provide new insights into genetic etiology of SCZ. Second, despite the fact that a recent GWAS metaanalysis in populations of East Asian ancestry (EAS) revealed comparative genetic architecture of SCZ between populations of European ancestry (EUR) and East Asian ancestry (EAS) (genetic correlation between EAS and EUR is $0.98 \pm 0.03$ ), this study also showed populationspecific associations [24]. For example, Lam et al. found that a large proportion of genome-wide significant variants identified in EAS showed dramatic differences in allelic frequency between EAS and EUR [24], further indicating the importance of conducting GWAS in nonEuropean populations. Third, accumulating data suggest that a large proportion of risk variants contribute to SCZ through modulating gene expression [29-31]. Therefore, it is important to pinpoint the potential target genes of the identified risk variants. To address these challenges, we firstly conducted a GWAS in Han Chinese population $(N=8202)$. We then performed a largescale meta-analysis (a total of 143,438 subjects) through combining our results with summary statistics from previous GWASs conducted in EAS and EUR (i.e., summary statistics-based meta-analysis, fixed-effect model was used) [24]. We also performed a transcriptome-wide association study (TWAS) to pinpoint the potential target genes of the identified risk variants and explored the potential tissue and cell type that the identified risk variants and genes may exert their biological effects.

\section{Methods}

\section{Study subjects}

SCZ cases were from inpatient and outpatient services of collaborating mental health centers of China. Part of cases has been described in our previous studies for candidate gene analyses [32-34]. Diagnosis was based on Diagnostic and Statistical Manual of mental disorders (DSM-IV) criteria, with the use of Structured Clinical Interview for DSM-IV (SCID) Axis I Disorders. All relevant and detailed information (including onset of SCZ, first onset or remitted, symptoms and chief complaint, duration course, family history of psychiatric disorders, medication history) were carefully evaluated by at least two independent experienced psychiatrists to reach a consensus DSM-IV diagnosis. Detailed information about diagnosis had been described in previous studies $[33,34]$. The average age of cases and controls were $35.67 \pm 10.29$ and $28.82 \pm 6.83$ years, respectively. $37.45 \%$ cases and $54.53 \%$ controls were males, respectively. All participants provided written informed consents. This study was approved by the Ethical Committee and internal review board of the Kunming Institute of Zoology (No: SMKX-20191215-07) and participating hospitals and universities (including the Second Affiliated Hospital of Xinxiang Medical University and Xi'an Jiaotong University). The samples were recruited from 2010 to 2018.

\section{DNA extraction and genotyping}

Genomic DNA was extracted from the peripheral blood with the use of QIAamp DNA blood mini kit (Cat. No: 51106). We used two types of genotyping platforms (arrays), including Illumina ASA (BeadChip Array Asian Screening Array-24+v1.0 HTS ASAMD-24v1-0) and GSA (BeadChip Array Global Screening Array-24+v2.0 HTS GSA v2.0+Multi-Disease). For ASA array (including 743,722 variants), $56.7 \%$ of the variants are common variants (with minor allele frequency $>0.05$ ), 30.8\% are low-frequency variant (with minor allele frequency between 0.01 and 0.05 ), and $12.5 \%$ are rare variants (minor allele frequency <0.01). ASA array includes a broad spectrum of pharmacogenomics markers $(N=5588)$ obtained from CPIC guidelines (www.cpicpgx.org) and the PharmGKB database (www.pharmgkb.org). In addition, the ASA array contains about 50,000 SNPs selected from ClinVar database (www.ncbi.nlm.nih.gov/clinvar). For GSA array ( $N=759,993$ variants), $54.4 \%$ of the variants are common variants (with minor allele frequency > 0.05 ), $18.1 \%$ are low-frequency variant (with minor allele 
frequency between 0.01 and 0.05 ), and $27.4 \%$ are rare variants (minor allele frequency <0.01). Similar with ASA array, the GSA array includes multiple expert curated variants obtained from ClinVar (www.ncbi.nlm. nih.gov/clinvar), PharmGKB (www.pharmgkb.org), NHGRI (www.genome.gov/), and other databases. More details about the ASA and GSA arrays can be found in the official Illumina website (https://www.illumina.com/ products/by-type/microarray-kits). Genotyping assays were conducted at Guoke Biotechnology Co., LTD in Beijing (www.bioguoke.com).

\section{Quality control}

We conducted quality control as previously described $[23,24,35]$, with some minor revisions. The main consideration is to include more subjects and variants under the premise of ensuring data quality. The individuallevel quality control (QC) was processed as follows: (1) Samples with genotype missing rate $>0.03$ were excluded; (2) Samples with a heterozygosity (calculated by plink 1.9 -het command) deviated \pm 3 stand deviation (s.d.) from the mean heterozygosity of all samples were excluded; (3) Related samples were inferred by KING software (http://people.virginia.edu/ wc9c/KING/) [36]. We used -related command implemented in KING to infer the potential kinship coefficients with 3rd degree. After inferring relatedness between samples, KING program exported related and unrelated samples. Related samples were then excluded. (4) We checked the sex of samples by using Plink (v1.09) (with --check-sex command). Samples with inconsistent sex information were excluded. We excluded samples if their sex estimated by genotype data and medical record information were inconsistent. In addition, we also excluded samples whose sex could not be accurately predicted based on the genotype data.

The SNP-level QC are as follows: (1) Excluding SNPs with a call rate $<97 \%$; (2) As described in the study of Lam et al. [24], we excluded SNPs with a significant deviation from Hardy-Weinberg equilibrium $(P<1.0 \times$ $10^{-6}$ in controls and $P<1.0 \times 10^{-10}$ in cases); (3) Excluding SNPs with a minor allele frequency (MAF) < 0.01; (4) Only biallelic SNPs were retained for further analysis. The QCs were performed by Plink 1.9 software [37]. Our analysis started in March 2020 and finished in May 2021.

\section{Principal component analysis (PCA)}

We performed principal component analysis (PCA) to assess population stratification and exclude the outliers. The subjects from the 1000 Genomes project (including CHB (Han Chinese in Beijing, China), CHS (Southern Han Chinese), JPT (Japanese in Tokyo, Japan), YRI (Yoruba in Ibadan, Nigeria), and CEU (Utah residents with northern and western European ancestry)) [38] were used as references and PCA was performed with GCTA software [39]. Samples that were not clustered with subjects of Han Chinese ancestry were excluded. We then used the Smartpca program to calculate principal components as covariates to correct potential population stratification [40, 41]. We excluded the MHC region (chr6: 25-34 MB) when performing PCA. Five PCA iterations were run and top 20 principal components (PCs) were calculated. Samples that were away from 6 standard deviations (s.d.) of the mean of each PC were excluded. After stringent quality control, 3493 cases and 4709 controls were retained for GWAS.

For each group (ASA subgroup 1 and subgroup 2, GSA), the top 20 PCs were calculated. The PCs were calculated as covariates to correct potential population stratification for each group (ASA subgroup 1 and subgroup 2, GSA), respectively. As suggested by Price et al. [41], we included a variable number of PCs as covariates to perform logistic regression analysis in each group (ASA subgroup1, ASA subgroup2, GSA). We used genomic control inflation factor $\left(\lambda_{\mathrm{GC}}\right)$ to estimate the effect of the number of PCs on population stratification adjustment $[41,42]$. The selection of the final number of PCs was mainly based on the following criteria [43]: (1) the $\lambda_{\mathrm{GC}}$ should as close to 1 as possible (indicating population stratification is well controlled); (2) when including more PCs, the $\lambda_{\mathrm{GC}}$ did not change significantly (indicating that the number of PCs selected is enough to adjust population stratification). Based on these criteria, we finally selected $10 \mathrm{PCs}$ for ASA subgroup 1, 4 PCs for ASA subgroup 2, and 12 PCs for GSA samples respectively.

\section{Genotype imputation}

The imputation of the genotypes was performed by Eagle [44] and minimac3 [45]. The Eagle was used for phasing the genotype data of each chromosome. The reference panel from the 1000 Genomes project (Phase 3) [38] was downloaded from the minimac3 website (https://genome.sph.umich.edu/wiki/Minimac3). The imputed data were further processed with the following QC steps: (1) SNPs with an imputation quality score < 0.8 were excluded; (2) SNPs with a MAF $<0.01$ were excluded; (3) SNPs that significantly deviated from the Hardy-Weinberg equilibrium $\left(P<1 \times 10^{-6}\right.$ in controls, $P<1 \times 10^{-10}$ in cases) were excluded; (4) Only SNPs with a genotype imputation rate $>97 \%$ were remained; (5) Only biallelic SNPs were included for further association analysis. After QC, the total number of SNPs included in GWAS analysis was 4,724,225 (for ASA arrays) and 5,107,135 (for GSA arrays). The number of overlapping SNPs between the two platforms is 3,937, 527. To make our QC procedures more clear and easy 
to follow, we provided the detailed information about QC (to list all pre- and post-imputation QC steps with excluded and remaining SNPs and participants after each step) in Additional file 1: Figure S1.

\section{GWAS and meta-analysis}

As our samples were genotyped with Illumina ASA and GSA Chip arrays, to avoid potential effects of different array platforms on our analysis, we performed genomewide association analysis separately. We firstly performed genetic association analysis in our samples (ASA subgroup 1, ASA subgroup 2, GSA, respectively) by using logistic regression. This association analysis was based on genotype data of each group (i.e., ASA subgroup 1, ASA subgroup 2, GSA), with PCs included as covariate. The results from these three genome-wide summary statistics were then meta-analyzed (summary statistics-based meta-analysis, fixed-effect model was used). GWAS were performed by Plink (v1.09) software [37]. We further performed a summary statistics-based meta-analysis in East Asian populations through combining the results of our study and GWAS summary statistics (only EAS were used) from a recent study by Lam et al. (including 22,778 case and 35,362 controls) [24]. Finally, we carried out a trans-ancestry meta-analysis (summary statistics-based, fixed effect model) through combining our results with the summary statistics from East Asians and Europeans (containing 56,418 cases and 78,818 controls) $[24,26]$.

\section{Defining of independent risk loci and new loci identification}

To identify independent risk loci, we used FUMA [46] to clump the association results (with the default parameters). Different reference panels were used for clumping. For the meta-analysis in EAS, subjects of East Asian ancestry from the 1000 Genomes project [38] were used. For the meta-analysis of EAS and PGC2 [24], subjects of European ancestry from the 1000 Genomes project [38] were used. The clumping processes were as follows: The minimum $r^{2}$ threshold for independent significant SNPs was set to 0.6 , which was used to define the boundaries of the genomic risk locus. When independent significant SNPs were defined in a locus, these SNPs were further processed to identify the lead SNP for each locus. The minimum $r^{2}$ for defining lead SNPs was set to $r^{2}=0.1$. The LD blocks of independent significant SNPs that $<$ $250 \mathrm{~kb}$ were merged into a single genome locus. More details about LD clump can be found in FUMA website (https://fuma.ctglab.nl/tutorial). Visualization of association results of interest SNPs and its nearby variants were generated with locuszoom [47] (http://locuszoom. org/genform.php?type=yourdata).
We compared the genome-wide significant (GWS) loci identified in this study and published SCZ GWAS $[21,23,24,26]$, and we also included GWASs listed in GWAS catalog database in FUMA [46]. We used following approaches for risk loci comparison: (1) If the published SCZ GWAS provide GWS index SNPs and its corresponding genomic region (chromosomal coordinates), we overlapped genomic region of our GWS loci with these published risk loci. Nonoverlapping loci were defined as new loci; (2) If the published SCZ GWAS only list GWS index SNPs, the newly identified risk loci (by us) should have no overlap with these GWS index SNPs, the index SNPs of the newly identified risk loci (by us) should also not be in linkage disequilibrium with the reported GWS index SNPs $\left(\mathrm{R}^{2}<0.1\right)$; (3) GWAS catalog database implemented in FUMA [46] were also used to identify new SCZ GWS loci.

\section{Polygenetic risk score profiling}

Polygenetic risk score profiling was performed by PRSice2 [48]. The PRS scores derived from training datasets were used to predict the case-control status of our samples. And the samples included in this study were used as target sample. We used classic clumping and threshold method. We run PRS using default parameters in PRSice2 [48]. The detailed clumping parameters of PRS calculation were as follows: --clump-kb 250, --clump-p 1.0, --clump-r2 0.10. Summary statistics from EAS [24], PGC2 EUR [24], EAS + EUR [24], and CLOZUK+PGC2 [21] were used as training datasets in PRS analysis. And we set $10 P$ value thresholds $\left(5 \times 10^{-8}, 5 \times\right.$ $\left.10^{-5}, 0.0001,0.001,0.01,0.05,0.1,0.2,0.5,1\right)$ to analyze the phenotypic variance explained at different $P$ value cutoffs.

\section{Tissue and cell-type enrichment analysis}

To explore if schizophrenia associations were enriched in specific human tissues, we performed tissue enrichment analysis by using MAGMA (Version 1.08) [49], which is implemented in FUMA software (Version 1.3.6a) [46]. Briefly, gene expression data of different human tissues (RNA sequencing data from the Genotype-Tissue Expression (GTEx) [50] consortium) were used to identify the genes that differentially expressed in a specific tissue. Based on the GWAS $P$ values, MAGMA quantifies the degree of association between a gene and schizophrenia (i.e., obtain a gene-level $P$ value) by using a multiple linear principal component regression model. MAGMA then tests if schizophrenia associations were enriched in the specifically expressed genes in a specific tissue. More detailed information about tissue enrichment 
analysis can be found in FUMA website (https://fuma. ctglab.nl/).

Cell-type enrichment analysis was also performed using MAGMA (Version 1.08) [49]. Single-cell RNA sequencing data from the mouse central nervous system (CNS) [51] were downloaded and processed as described in Bryois et al. [52]. Briefly, a total of 160, 769 high-quality single-cell RNA-seq data were analyzed. Human genes were mapped to orthologous mouse genes (one to one) based on MGI annotations (http://www.informatics.jax.org/homology.shtml), and genes that were not expressed in the mouse central nervous system (CNS) were excluded. The expression specificity of a specific gene was calculated as described in Bryois et al. [52] and the top 10\% that were most specifically expressed genes in each cell type were used for enrichment analysis. The $P$ values of MAGMA enrichment analysis were corrected by false discovery rate (FDR).

\section{Gene set enrichment analysis}

Gene set analysis was performed with MAGMA software (Version 1.08) [49]. MAGMA gene set analysis includes two main procedures. Firstly, by using the GWAS summary statistics and gene annotation files (NCBI b37, downloaded from official MAGMA website (https://ctg. cncr.nl/software/magma)), MAGMA maps SNPs to genes (with gene boundaries setting parameters "--annotate window $=35,10^{\prime \prime}$. MAGMA then calculates the association strength between a specific gene and schizophrenia. A gene and phenotype association matrix was generated in this step with default "snp-wise = mean" model. The MHC region (from 25 to $34 \mathrm{Mb}$ ) on chromosome 6 was excluded in analysis. Secondly, gene set analysis which was also known as competitive gene set analysis was performed. MAGMA tests whether the association of a target gene set with phenotype is greater than other genes that are not included in the gene set. The $P$ value of competitive gene set was used to determine the significance level. We downloaded gene sets from MSigDB database (v7.1) (http://software. broadinstitute.org/gsea/msigdb/) [53] and all GO terms (including cellular component, biological processes, and molecular functions). KEGG pathway gene sets were also included and a total of 10,378 gene set terms were compiled. We further retained 6194 gene sets with a gene number range from 10 to 200 for the final analysis. The final $P$ values (i.e., the association strength between gene sets and schizophrenia) of MAGMA competitive gene sets were corrected by false discovery rate (FDR).

\section{LD score regression analysis}

We conducted stratified linkage disequilibrium score regression (LDSC) [54] to partition SCZ SNP heritability and test enrichment of SCZ heritability in different functional annotations [54]. In addition, we also performed genetic correlation analysis between our Chinses cohort and the reported EAS samples (based on summary statistics) using LDSC [54].

\section{Transcriptome-wide association analysis}

We performed TWAS using the summary statistics from all combined samples (including our Han Chinese cohort, EAS and PGC2 [24], a total of 59,911 cases and 83, 527 controls) and brain eQTL (gene expression SNP weights) from the CommonMind Consortium (CMC, $N$ = 452) [55]. CMC measured gene expression in the dorsolateral prefrontal cortex (DLPFC) of human brain with the use of RNA sequencing. The CMC gene expression SNP weights were derived from the FUSION pipeline (http://gusevlab.org/projects/fusion/). Detailed information about sample collection, RNA extraction and sequencing, genotyping and statistical analysis of CMC dataset can be found in the original studies [55]. TWAS was performed on autosomal chromosomes using the FUSION.assoc.test.R script across all predictive models, such as LASSO, GBLUP, Elastic Net, and BSLMM. To determine which is the best model for TWAS, FUSION performed a five-fold cross-validating of each model. TWAS associations (i.e., genes) were considered "transcriptome-wide significant" if they passed a strict Bonferroni-corrected threshold for all genes tested in the dataset (corrected significance $P$ value: $0.05 / 3551=1.41 \times 10^{-5}$ ). Detailed description of the principle of FUSION and statistical model can be found in the original paper [56].

\section{Expression quantitative trait locus (eQTL) analysis}

We performed eQTL analysis using 4 public available brain eQTL resources, including the CommonMind Consortium (CMC) [55], LIBD BrainSeq Phase II RNAseq Project (LIBD2) [57], xQTL [58], and GTEx [50] project. Please refer to the original papers $[50,55,57$, 58] about the sample collection, RNA-seq data processing, and eQTL calculation.

\section{Differential expression analysis in schizophrenia cases and controls}

We examined the expression level of genome-wide significant genes in brains of SCZ cases $(n=559)$ and controls $(n=936)$ by using the PsychEncode data [59]. Briefly, brain gene expression data of 559 schizophrenia cases and 936 controls were quantified and analyzed by the PsychEncode. Detailed information on tissue collection, RNA sequencing, gene expression quantification, and differential expression analysis were provided in PsychEncode papers [59] and website (http: http:// resource.psychencode.org/). 


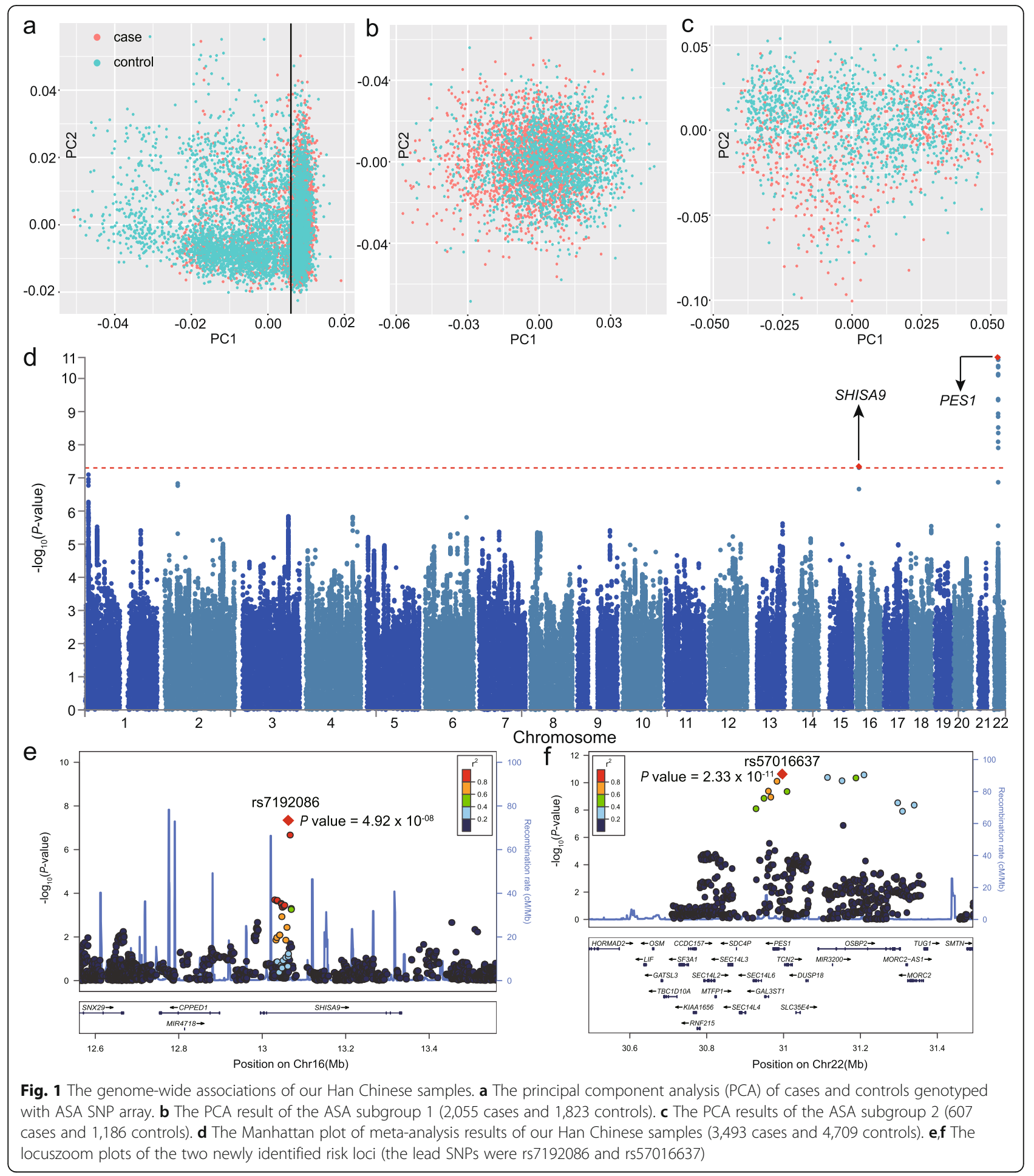

\section{Results}

\section{GWAS of Han Chinese cohort identified 2 new} schizophrenia risk loci

Our samples had no overlap with the previously published SCZ GWAS of Han Chinese population [17, 19, $23,60]$. We first performed a PCA analysis using the samples genotyped with Illumina Asian Screening Arrays (ASA) and found population stratification of our samples (Fig. 1a); we thus divided our samples into two genetically matched subgroups. After stringent QC and excluding outliers, 2055 cases and 1823 controls were included in subgroup 1, and 607 cases and 1186 controls were 
included in subgroup 2 (Fig. 1b, c). For samples genotyped with GSA arrays, no obvious population stratification was observed. After strict QC, the final samples genotyped with GSA arrays included in this study were 831 cases and 1700 controls (Additional file 1: Figure S2). PCA analysis using genotype data of our samples and the 1000 Genomes project [38] showed that all of our samples clustered with samples of Han Chinese ancestry (Additional file 1: Figures S3, S4). After strict QC, imputation using the 1000 Genomes project phase 3 panel [45] and post-imputation QC, a total of 3,937,527 biallelic SNPs from 3493 SCZ cases and 4709 healthy controls were retained for GWAS. Principal components (PCs) were included as covariates [40, 41] (10 PCs for ASA subgroup1, 4 PCs for ASA subgroup2, 12 PCs for GSA samples) when performing GWAS. We firstly meta-analyzed the samples genotyped with ASA arrays (subgroups 1 and 2). We then conducted a meta-analysis through combining the results from ASA and GSA arrays with the fixed effect model. The genomic inflation $\left(\lambda_{\mathrm{GC}}\right)$ of our combined meta-analysis (including ASA and GSA samples) was 1.10 , and the $\lambda_{1000}$ (scaled to a sample size of 1000 cases and 1000 controls) was 1.02 (which was very close to the reported values in previous Chinese GWAS [17, 23], 1.02 in Li et al. [23] and 1.019 in Yu et al. [17]) (Additional file 1: Figure S5), indicating that population stratification unlikely confounds our genome-wide association results.

Two genome-wide significant risk loci were identified in our sample (Fig. 1d). The lead risk SNP for the first locus is rs7192086 $\left(P=4.92 \times 10^{-08}, \mathrm{OR}=1.22\right)$, which is located in the intron 2 of the SHISA9 gene (Fig. 1e). Of note, a previous study (7308 cases and 12,834 controls) showed nominal association between rs7192086 and SCZ $\left(P=1.34 \times 10^{-05}, \mathrm{OR}=1.12\right)$ in European population [22]. The lead SNP for the second risk locus

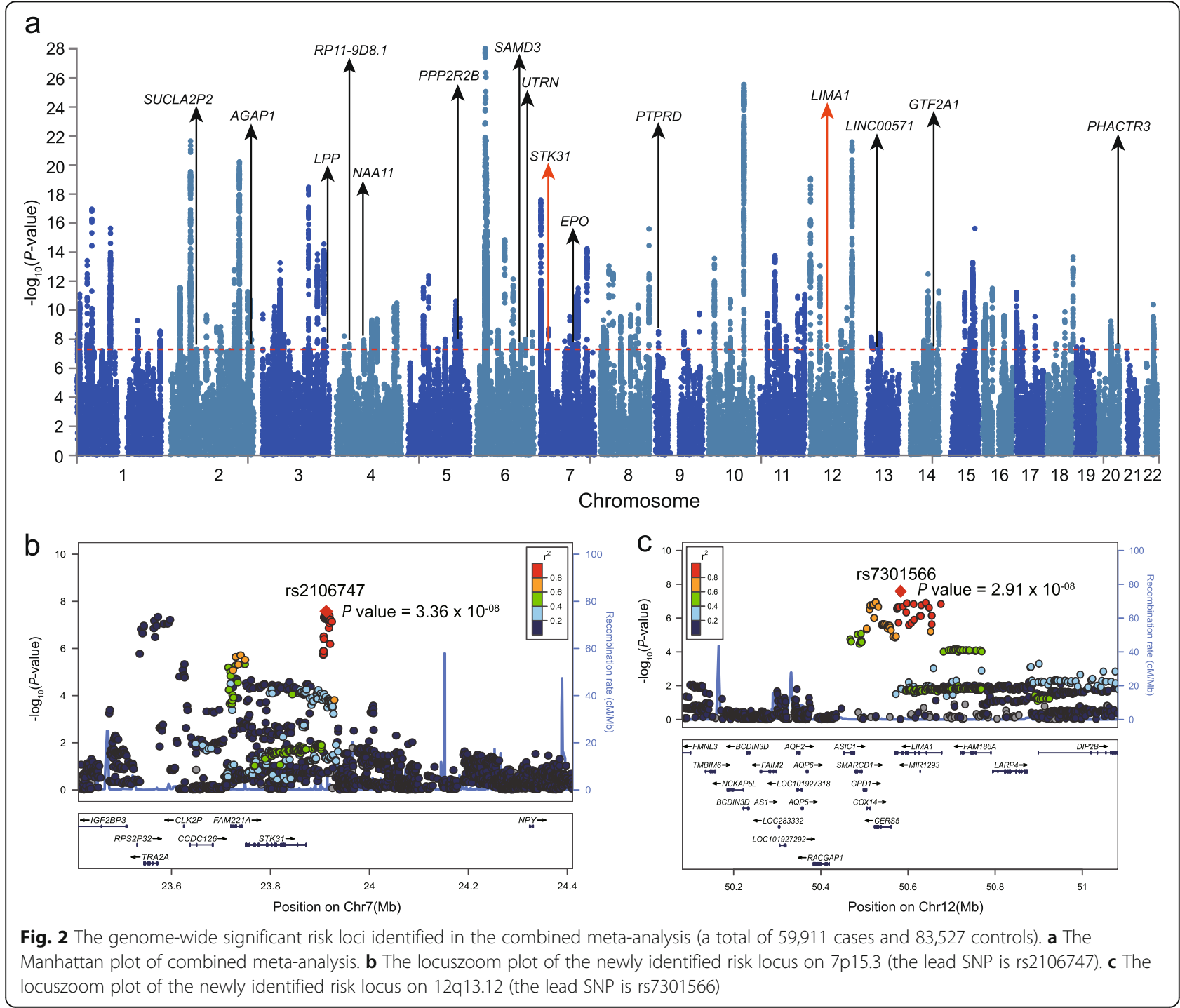


is $\operatorname{rs} 57016637\left(P=2.33 \times 10^{-11}, \mathrm{OR}=1.34\right)$ located in the intron 2 of PES1 (Fig. 1f). This genomic loci contain three independent significant SNPs, and the other two SNPs are rs117961127 $\left(P=2.73 \times 10^{-11}, \mathrm{OR}=1.35\right)($ located in the intron 2 of OSBP2) and rs116976860 (1.42 $\times 10^{-09}, \mathrm{OR}=1.33$ ) (located in the intergenic region of SEC14L6 and GAL3ST1). Of note, rs57016637 is an East Asian-specific polymorphism (Additional file 1: Figure S6).

\section{Meta-analysis with EAS and EUR identified 15 new risk loci}

We then carried out a meta-analysis through metaanalyzing GWAS data obtained from our sample and a recent GWAS conducted in EAS [24]. Our meta-analysis using combined EAS samples (26,271 cases, 40,071 controls) [24] identified 24 risk loci (Additional file 1: Figure S7). However, all of these loci have been reported in a previous study [24]. Intriguingly, we noticed that rs3845188 $\left(P=6.50 \times 10^{-08}, \mathrm{OR}=0.91\right)$ (which is located in the intron 1 of the $N E B L$ gene) reached the suggestive significance level $\left(P=5.00 \times 10^{-06}\right)$, suggesting this locus may be associated with SCZ (Additional file 1: Figure S8).

We further performed a meta-analysis through combining results of our study and GWAS results from EAS and PGC2 European samples (59,911 cases and 83,527 controls) [24]. We identified 153 genome-wide

Table 1 New genome-wide significant loci identified in this study (a total of 17 novel risk loci)

\begin{tabular}{|c|c|c|c|c|c|c|c|c|c|}
\hline $\begin{array}{l}\text { Genomic } \\
\text { locus }^{\mathrm{a}}\end{array}$ & Lead SNP & Chr & Pos & $\begin{array}{l}\mathrm{A} 1 / \\
\mathrm{A} 2\left(\mathrm{MA}^{\mathrm{b}}\right)\end{array}$ & $\begin{array}{l}\mathrm{MAF}_{-} \\
\mathrm{A}^{\mathrm{c}}\end{array}$ & $\begin{array}{l}\text { MAF }_{-} \\
U^{d}\end{array}$ & $\mathbf{P}$ & $\mathrm{OR}^{\mathrm{e}}$ & Nearby gene $(s)^{f}$ \\
\hline 1 & rs7192086 & 16 & 13061611 & $T / A(T)$ & 0.32 & 0.28 & $\begin{array}{l}4.92 \\
\times \\
10^{-08}\end{array}$ & 1.22 & SHISA9 \\
\hline 2 & rs57016637 & 22 & 30992925 & $\mathrm{G} / \mathrm{C}(\mathrm{G})$ & 0.20 & 0.16 & $\begin{array}{l}2.33 \\
\times \\
10^{-11}\end{array}$ & 1.34 & $\begin{array}{l}\text { SF3A1, CCDC157, RNF215, SEC14L2, MTFP1, SEC14L3, } \\
\text { SEC14L6, GAL3ST1, PES1, TCN2, SLC35E4, DUSP18, OSBP2, } \\
\text { MORC2 }\end{array}$ \\
\hline 3 & rs115487049 & 2 & 76297343 & $A / T(A)$ & 0.042 & 0.036 & $\begin{array}{l}4.72 \times \\
10^{-08}\end{array}$ & 1.07 & SUCLA2P2 \\
\hline 4 & rs10178509 & 2 & 236792838 & $\mathrm{~T} / \mathrm{C}(\mathrm{T})$ & 0.39 & 0.42 & $\begin{array}{l}1.08 \times \\
10^{-08}\end{array}$ & 0.95 & $A G A P 1$ \\
\hline 5 & rs1426271 & 3 & 188187250 & $A / G(G)$ & 0.35 & 0.33 & $\begin{array}{l}3.23 \times \\
10^{-08}\end{array}$ & 0.95 & $\angle P P$ \\
\hline 6 & rs2911914 & 4 & 37820679 & $\mathrm{~T} / \mathrm{G}(\mathrm{T})$ & 0.25 & 0.27 & $\begin{array}{l}2.12 \times \\
10^{-08}\end{array}$ & 0.95 & PGM2 \\
\hline 7 & rs6848123 & 4 & 80203425 & $A / C(C)$ & 0.14 & 0.16 & $\begin{array}{l}1.17 \times \\
10^{-08}\end{array}$ & 1.05 & NAA11 \\
\hline 8 & rs319227 & 5 & 146245762 & $A / C(A)$ & 0.33 & 0.30 & $\begin{array}{l}6.11 \times \\
10^{-09}\end{array}$ & 1.05 & $P P P 2 R 2 B$ \\
\hline 9 & rs12202107 & 6 & 130598623 & $\mathrm{~T} / \mathrm{C}(\mathrm{C})$ & 0.42 & 0.45 & $\begin{array}{l}2.51 \times \\
10^{-08}\end{array}$ & 1.05 & SAMD3,TMEM200A \\
\hline 10 & rs9386072 & 6 & 144859509 & $\mathrm{~A} / \mathrm{T}(\mathrm{A})$ & 0.43 & 0.47 & $\begin{array}{l}1.01 \times \\
10^{-08}\end{array}$ & 0.93 & UTRN \\
\hline 11 & rs2106747 & 7 & 23911499 & $A / G(G)$ & 0.40 & 0.42 & $\begin{array}{l}3.36 \times \\
10^{-08}\end{array}$ & 1.05 & TRA2A, FAM221A, STK31 \\
\hline 12 & rs492430 & 7 & 100313099 & $\mathrm{~T} / \mathrm{G}(\mathrm{T})$ & 0.065 & 0.058 & $\begin{array}{l}2.26 \times \\
10^{-08}\end{array}$ & 1.07 & ACTL6B, GNB2, GIGYF1, POP7, EPO \\
\hline 13 & rs59761926 & 9 & 10238178 & $\mathrm{~T} / \mathrm{C}(\mathrm{T})$ & 0.37 & 0.39 & $\begin{array}{l}3.02 \times \\
10^{-09}\end{array}$ & 0.95 & PTPRD \\
\hline 14 & rs7301566 & 12 & 50581647 & $\mathrm{~T} / \mathrm{C}(\mathrm{T})$ & 0.099 & 0.091 & $\begin{array}{l}2.91 \times \\
10^{-08}\end{array}$ & 1.06 & ASIC1, SMARCD1, GPD1, COX14, CERS5, LIMA1 \\
\hline 15 & rs6563592 & 13 & 38814939 & $\mathrm{~T} / \mathrm{G}(\mathrm{G})$ & 0.31 & 0.30 & $\begin{array}{l}2.13 \times \\
10^{-08}\end{array}$ & 0.95 & LINC00571 \\
\hline 16 & rs2099108 & 14 & 81675420 & $C / G(G)$ & 0.45 & 0.47 & $\begin{array}{l}2.67 \times \\
10^{-08}\end{array}$ & 1.05 & GTF2A1,STON2 \\
\hline 17 & rs6100546 & 20 & 58252407 & $\mathrm{~T} / \mathrm{C}(\mathrm{C})$ & 0.079 & 0.076 & $\begin{array}{l}2.79 \times \\
10^{-08}\end{array}$ & 0.94 & PHACTR3 \\
\hline
\end{tabular}

${ }^{a}$ The new loci identified in our Han Chinese cohort are shown in bold. ${ }^{b}$ minor allele of our Chinese samples. ${ }^{c}$ minor allele frequency in our Chinese cases. ${ }^{d}$ minor allele frequency in our Chinese controls. ${ }^{e}$ Odds ratio is based on A1; ${ }^{f}$ The nearby genes were defined with FUMA. FUMA firstly identified the SNPs that were in LD $\left(r^{2}>0.6\right)$ with independent lead SNPs. And these SNPs were further used to identify the nearby genes by FUMA with default parameters (posMapWindowSize $=10 \mathrm{~kb}$ ). More details about defining nearby genes can be found in FUMA website: https://fuma.ctglab.nl/tutorial\#parameters 
independent risk loci in the combined samples (Fig. 2a) (The definition of independent risk loci was described in methods section). Among these 153 risk loci, 15 were novel (Table 1, Additional file 1: Table S1). In total, we identified 17 new risk loci for SCZ.

\section{Identification of potential target genes of the newly identified risk SNPs}

To identify the potential target genes of the newly identified risk SNPs, we performed eQTL analysis using data from the human brain tissues. Among the 17 lead SNPs, 9 showed associations (uncorrected $P<0.05$ ) with expression of 33 genes in the human brain (Additional file 1: Table S2). Of note, $r$ s2106747 $\left(P=3.36 \times 10^{-08}, \mathrm{OR}=\right.$ 1.05 , Fig. 2b) showed a strong association with FAM221A expression in all four eQTL datasets. Another interesting SNP is $\operatorname{rs7301566}\left(P=2.91 \times 10^{-08}, \mathrm{OR}=\right.$ 1.06, Fig. 2c), which was associated with expression of several genes, including COX14, DIP2B, CERS5, RP460503.4, SPATS2, ASIC1, and ATF1 (Additional file 1: Table S2). These results suggest that the newly identified risk variants may contribute to $\mathrm{SCZ}$ risk through regulating expression of these eQTL genes.

\section{Differential expression analysis in schizophrenia cases and controls}

We explored the expression level of potential target genes of the newly identified risk variants in SCZ cases and controls using expression data from the PsychEncode (including 559 cases and 936 controls) [59]. Among the 33 potential target genes, 14 showed nominal difference in expression in SCZ cases compared with controls (Additional file 1: Table S3), supporting that the newly identified risk variants may confer SCZ risk by regulating the expression of these target genes. In addition, we also examined the expression of genes located near the two newly identified loci in our Chinese samples (Table 1, Fig. 1e, f). We found that SHISA9 showed a trend of upregulation in brains of $\mathrm{SCZ}$ cases compared with controls $(P=0.053)$. Interestingly, OSBP2 was significantly downregulated in brains of SCZ cases compared to controls $\left(P=1.07 \times 10^{-07}\right)$. Taken together, these expression data provide further evidence that support the newly identified risk variants may confer risk of SCZ through modulating expression level of these genes.

\section{Polygenic risk score (PRS) profiling}

We conducted PRS analysis to predict the case-control status of our samples (ASA subgroup 1) and estimate the phenotypic variance (of our samples) that can be explained by the published GWAS summary statistics data [24]. When using the summary statistics from EAS as training set [24], the explained variance (estimated by Nagelkerke $\mathrm{R}^{2}$ ) ranged from 0.4 to $5.9 \%$ and the training data has the largest variance explanation at $P$ value $=0.2$ $\left(P=2.19 \times 10^{-37}\right)$ (Fig. 3). The training set from EAS + EUR has an overall better prediction performance and variance explanation at each $P$ value threshold than EAS [24], and the explained variance ranged from $2.0 \%$ to $6.5 \%$. At $P$ value $=0.01$ threshold, the training dataset has the largest variance explanation $\left(P=4.69 \times 10^{-41}\right)$. The EUR and CLOZUK+PGC2 training sets had relatively poor prediction performance than the EAS and EAS + EUR training set. The explained variance (estimated by Nagelkerke $\mathrm{R}^{2}$ ) ranged from 0.6 to $2.7 \%$ and 0.7 to $4.4 \%$ for EUR and CLOZUK+PGC2 training datasets, respectively. The PRS analysis indicated that EAS and EAS + EUR training sets had relative good power to predict the SCZ and healthy controls status of our sample, and the EAS + EUR training set had better prediction performance.

\section{Identification of tissues and cell types associated with schizophrenia}

We conducted tissue enrichment analysis by using GWAS associations from the combined samples (i.e., our cohort, EAS and PGC2 EUR [24]) and gene expression from GTEx [50], with the use of FUMA [46]. SCZ heritability was mainly enriched in brain tissues (Additional file 1: Figure S9a). Of note, two brain cerebellum tissues showed the strongest associations (brain cerebellar hemisphere, $P=2.54 \times 10^{-23}$, and brain cerebellum, $\left.P=8.51 \times 10^{-23}\right)$. The frontal cortex (BA9) $(P=1.28 \times$ $\left.10^{-19}\right)$ and brain cortex $\left(P=2.20 \times 10^{-18}\right)$ also showed significant enrichment.

We further conducted cell-type enrichment analysis (Additional file 1: Figure S9c). Similar with previous findings [52], two telencephalon neuronal cell types, including telencephalon projecting excitatory interneurons $\left(P=1.12 \times 10^{-09}, \mathrm{FDR}<0.05\right)$ telencephalon projecting inhibitory interneurons $\left(P=1.15 \times 10^{-06}, \mathrm{FDR}<0.05\right)$, showed significant enrichment for SCZ associations. We also identified other novel associations, including oligodendrocytes $\left(P=8.03 \times 10^{-07}, \mathrm{FDR}<0.05\right)$, cholinergic and monoaminergic neurons $\left(P=6.06 \times 10^{-05}\right.$, FDR $<$ $0.05)$, and cerebellum neurons $\left(P=4.99 \times 10^{-05}\right.$, FDR $<$ 0.05). Collectively, our results suggest that SCZ risk genes are actively expressed in these identified tissues and cell types, implying the pivotal roles of these tissues and cell types in SCZ.

\section{Gene set analysis identified enriched gene sets and pathways}

We carried out gene set enrichment analysis and identified 6 significant enriched terms, including neuron spine $\left(P=7.06 \times 10^{-07}, \quad\right.$ FDR $\left.=0.0044\right)$, membrane depolarization during action potential $\left(P=4.72 \times 10^{-06}\right.$, FDR $=0.015)$, cytosolic calcium ion transport $(P=4.03$ 


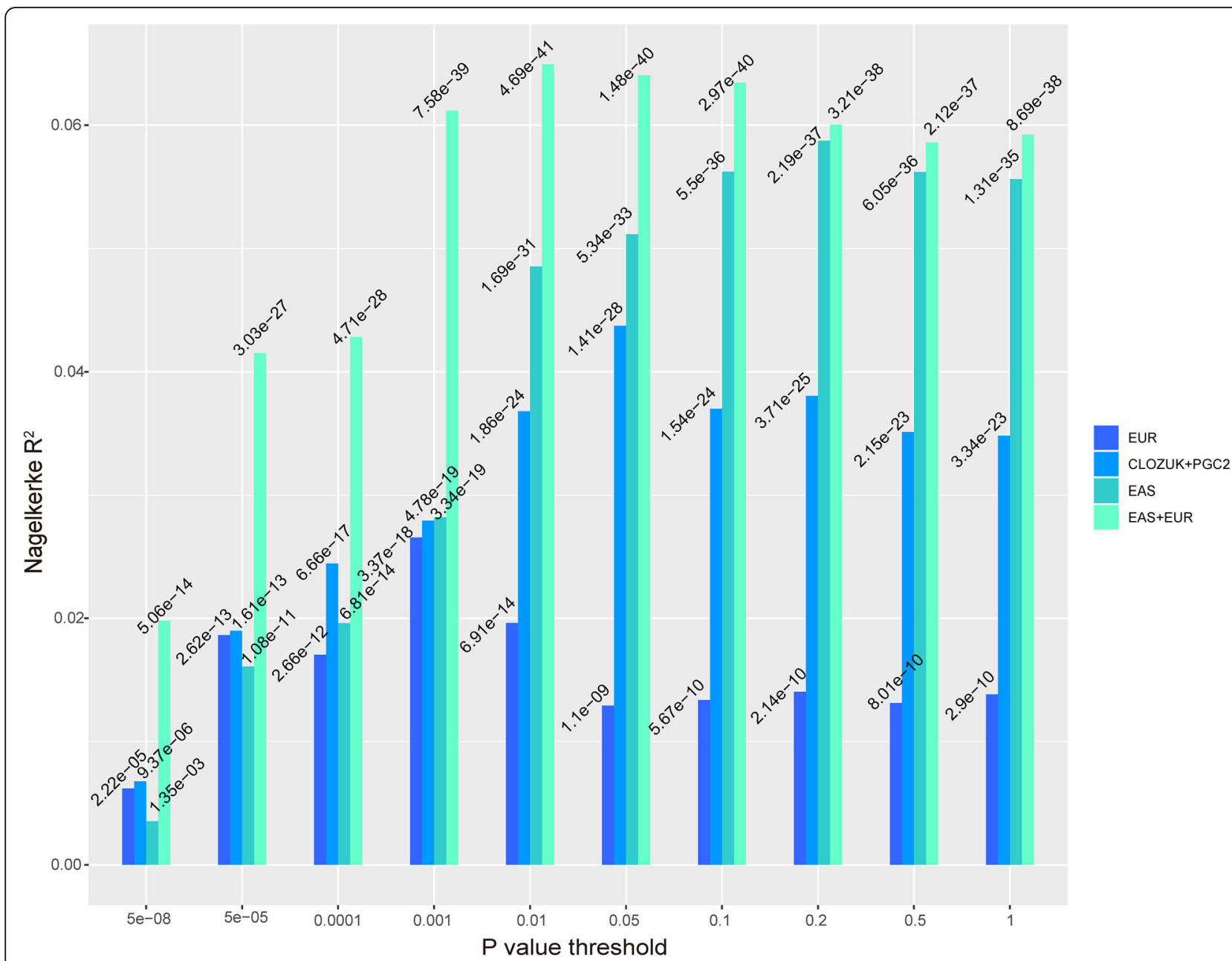

Fig. 3 PRS analysis results of our ASA subgroup 1 samples. PRSs were computed using GWAS summary statistics from four training sets. The first is EAS (EAS) training set (including 22,778 SCZ cases and 35,362 controls). The second is EAS + PGC2 European (EAS + EUR) training set (including 56,418 SCZ cases and 78,818 controls). The third is PGC2 European (EUR) training set (33,640 cases and 43,456 controls), and the fourth is CLOZUK+PGC2 (CLOZUK+PGC2) training set (40,675 SCZ cases and 64,643 controls). The trans-ancestry EAS + EUR data set had better performance at different $P$ thresholds

$\left.\times 10^{-05}, \mathrm{FDR}=0.045\right)$, voltage gated sodium channel complex $\left(P=4.09 \times 10^{-05}\right.$, FDR $\left.=0.045\right)$, $\mathrm{t}$ tubule $(P=$ $\left.4.32 \times 10^{-05}, \mathrm{FDR}=0.045\right)$, and voltage gated sodium channel activity $\left(P=2.57 \times 10^{-05}\right.$, FDR $\left.=0.045\right)$. In addition, a few gene sets also showed a trend of enrichment, including regulation of synaptic plasticity $(P=1.5 \times$ $\left.10^{-04}, \mathrm{FDR}=0.074\right)$ and neurotransmitter receptor complex $\left(P=2.00 \times 10^{-04}, \mathrm{FDR}=0.074\right)$ (Additional file 1 : Table S4).

\section{Enrichment of schizophrenia heritability in conserved genomic regions, promoters, and enhancers}

LDSC analysis showed that SCZ associations were mainly enriched in various conserved genomic regions, promoters, and enhancers (SuperEnhancer_Hnisz, H3K27ac_Hnisz) (Additional file 1: Figure S9b), which were consistent with previous findings $[26,54]$.

\section{TWAS analysis identified risk genes for schizophrenia}

We conducted a TWAS to identify genes whose cisregulated expression were associated with SCZ. We identified 76 transcriptome-wide significant genes (corrected by multiple comparison testing) (Fig. 4 and Additional file 1: Table S5). Among these identified genes, 24 have been reported in a previous study [59]. Of note, GIGYF1 $\left(P=3.03 \times 10^{-05}\right)$ (located near the newly identified risk loci) showed a trend of TWAS significance (Additional file 1: Table S5). Taken together, our TWAS identified 52 new risk genes whose cis-regulated expression change may have a role in SCZ.

\section{Discussion}

In this study, we first performed a GWAS for SCZ in Han Chinese samples. We then conducted metaanalyses by combining our results and the published 


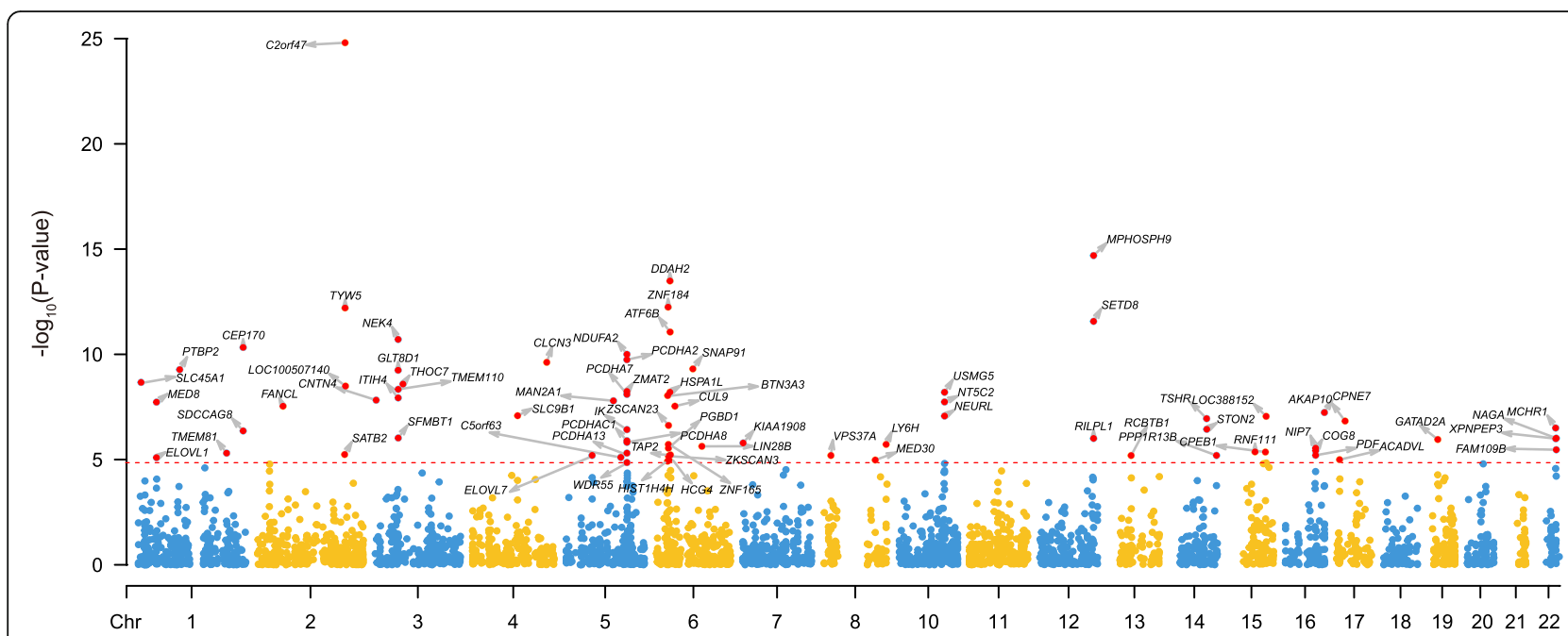

Fig. 4 TWAS results. The red line indicates the significant level corrected by the Bonferroni test. The summary associations from all combined samples (including this study, EAS and EUR) were used to perform TWAS

GWAS summary statistics from individuals of East Asian and European ancestry [24]. We identified 2 new genome-wide significant risk loci in our Han Chinese cohort. SNP rs7192086 was located in the intron 2 of the SHISA9 gene. SHISA9 (also known as CKAMP44) protein is a brain-specific type-I transmembrane protein and is highly expressed in hippocampal dentate gyrus and brain cerebral cortex [61]. SHISA9 is enriched at postsynaptic sites and its intracellular domain contains a PDZ domain interaction site which could physically interact with AMPA-type glutamate receptor (AMPAR); thus, SHISA9 plays important roles in synaptic shortterm plasticity [61]. Notably, the AMPAR shows abnormal forward trafficking in the frontal cortex of SCZ patients [62]. These lines of evidence suggest that SHISA9 may contribute to SCZ by affecting the function of AMPAR and synaptic transmission. However, further functional studies are warranted to reveal the role of SHISA9 in SCZ.

Another genome-wide significant risk variant identified in our sample is rs57016637. Intriguingly, we noticed that rs57016637 is fixed in other populations (Figure S8). Despite the fact that the majority of risk variants have similar effects between EUR and EAS populations [23], population heterogeneity still exists. For example, rs374528934 was reported to be strongly associated with SCZ in EAS $\left(P=5 \times 10^{-11}\right)$. Nevertheless, the MAF of rs374528934 in EUR is quite low (0.7\%) [24]. Our data suggest that rs57016637 may be a Han Chinese-specific risk variant for SCZ. SNP rs117961127 (in LD with lead SNP rs57016637 in the loci, $\mathrm{r}^{2}=0.32$ in 1000 East Asian samples) was located in the intron 2 of $O S B P 2$, a gene that encodes a cholesterol-binding protein. Cholesterol levels were reported to be altered in
SCZ cases compared to controls [63]. In addition, Krakowski et al. showed that cholesterol levels were strongly associated with cognition in SCZ [64]. These data suggest that OSBP2 may have a role in SCZ through regulating cholesterol levels. Further investigating the role of OSBP2 in SCZ is needed.

Two novel GWAS loci reported in our analysis did not reach GWS level in our follow-up meta-analysis with EAS and EAS + EUR samples (Additional file 1: Table S1). Of note, previous studies have also observed similar results in GWAS studies of Han Chinese [19, 60]. For example, the top associations identified by Shi et al. (rs16887244, rs10489202) [19] and Yue et al. (rs1233710, rs1635, rs2142731, rs11038167, rs11038172, rs835784) [60] in Chinese population did not reach genome-wide significance level in a larger meta-analysis (in EAS) reported by Lam et al. [24]. More work is needed to explore if this observation is due to population-specific associations or genetic heterogeneity between regional samples.

By meta-analyzing our results with GWAS associations from EAS and EUR [24], we identified 15 new risk loci, including 7p15.3 (the lead risk SNP is rs2106747, which was strongly associated with the expression level of FAM211A) and 12q13.12 (the lead risk SNP is rs7301566, which was an eQTL of several genes, including COX14, DIP2B, CERS5, RP4-605O3.4, SPATS2, $A S I C 1$, and $A T F 1$ ). These new risk loci provide valuable clues for further functional study. Further functional investigation of these risk genes will also provide important insights into SCZ pathogenesis and help to develop potential therapeutic targets. Some of our newly identified GWS loci are located in genomic regions near previous reported loci. For example, $\operatorname{rs319227}(P=6.11 \times$ 
$10^{-09}$, Table 1$)$ and $\operatorname{rs} 11958187\left(P=9.39 \times 10^{-09}\right.$, reported by Lam et al. [24]) are located near $P P P 2 R 2 B$, but these two index SNPs are not in LD $\left(\mathrm{R}^{2}<0.1\right)$. In addition, rs2106747 $\left(P=3.36 \times 10^{-08}\right.$, Table 1$)$ and rs112316332 $\left(P=3.04 \times 10^{-08}\right.$, reported by Lam et al. [24]) also showed similar results. These results suggest that these risk loci are genetically independent. However, more work is warranted to elucidate the functional mechanisms of these loci.

We compared our results with the findings reported by PGC3 (preprinted on medRxiv) [65]. We found that 6 loci (index SNPs are rs115487049, rs6848123, rs319227, rs59761926, rs7301566, rs6563592) reported in our study (Table 1) also show significant associations with SCZ in PGC3. This observation suggested though our sample size is relatively small, it could help us to discover new associations. A meta-analysis with PGC3 will help to identify more new associations and the underlying genetic basis of SCZ

Despite the fact that SCZ risk associations were highly shared between EAS and EUR, conducting GWAS in EAS is still important as it could improve our understanding of the underlying biology of SCZ. Firstly, Lam et al. showed that the genetic correlation between the EAS and EUR GWAS summary statistics is 0.98 , indicating that the genetic basis of SCZ are highly shared between EAS and EUR. With the increasing EAS sample, novel risk loci well be identified continuously, which will help us to understand the genetic basis of SCZ better. In addition, Lam et al. also reported EAS-specific association, e.g., $r$ 3745528934 ( $P=5$ $\times 10^{-11}$, minor allele frequency is 0.45 and 0.007 in EAS and EUR, respectively). These results demonstrated that the EAS GWAS summary statistics can not only facilitate to discover the genetic associations shared between EAS and EUR, but also help to identify EAS-specific GWAS associations. Finally, genome-wide associations from different populations help to improve fine-mapping [24]. We believe that EAS GWAS summary statistics will provide important insights into the genetic architecture (both shared with EUR population and EAS-specific) and the underlying biology of SCZ.

In the meta-analysis of EAS samples (our sample + EAS) [24], we found some novel loci (compared with EAS summary statistics alone). However, these loci also reached genome-wide significance level in EAS + PGC2 EUR summary statistics [24]. For example, rs12031518 reached genome-wide significance level in our EAS metaanalysis (our sample + EAS) $\left(P=4.96 \times 10^{-08}\right)$. Of note, this SNP did not reach genome-wide significance level in EAS samples $\left(P=7.58 \times 10^{-08}\right)$. However, it showed genome-wide significant association in EAS + PGC2 EUR summary statistics $\left(P=6.43 \times 10^{-11}\right)$ [24]. We calculated the genetic correlation between our ASA/GSA samples and the reported EAS (22,778 cases; 35,362 controls) [24]. Although these two GWAS summary statistics are highly correlated (the genetic correlation is 0.71 ), the genetic correlation is not very close to 1 . A possible reason is that the sample size included in our study is relatively small (3493 cases and 4709 controls) compared with the reported EAS samples (22,778 case and 35,362 controls).

PRS analysis revealed several interesting results. First, EAS training set had overall better performance than EUR and CLOZUK+PGC2 samples (though the sample size of EAS is less than the two GWAS summary statistics), indicating that similar ethnic background (of the EAS summary statistics) helps to improve the PRS prediction performance. Second, CLOZUK+PGC2 training set had better performance than EUR, indicating that the training set with larger sample size had better performance. Third, EAS + EUR GWAS summary statistics had the best performance than other training sets. This result reflects that trans-ancestry meta-analysis improves the prediction power.

Tissue and cell-type enrichment analysis revealed that SCZ associations showed the significant enrichment in the cerebellum, suggesting the potential role of cerebellum in SCZ. Of note, several previous studies also suggested that cerebellum may play an important role in SCZ [66-69]. These results suggest that the cerebellum may have a pivotal role in SCZ etiology.

Our study has several limitations. Firstly, our sample size is relatively small compared with recent SCZ GWAS cohort, such as PGC2 [26], Clozuk [21], or East Asian meta-analysis [24]. Additional SCZ risk loci will be found with the increase of sample size. Secondly, although we reported 17 novel risk loci, the casual variants and genes of these identified risk loci remain largely unknown. Further work, including pinpointing causal variants and genes, functional characterization of risk genes, exploring the role of risk genes in developing and adult brain, will provide pivotal insights into SCZ pathophysiology. Thirdly, we used eQTL data from Europeans to explore the associations between genome-wide significant SNPs and gene expression level in human brain. Considering that some novel risk loci were from our Chinese cohort, an ideal approach is to check the effect of the novel genetic variations and gene expression both in EAS and EUR populations. However, the brain eQTL data in EAS is not publically available so far. More work is needed to explore if these genetic variations also associated with gene expression in Chinese population. Fourthly, although including PCs as covariate is a regular and useful way to correct population stratification of GWAS, challenges remain in PCA. For example, selecting the optimal number of PCs [70] remains an open question (i.e., is relatively arbitrary, different numbers of PCs were reported in different studies). In addition, more work is needed to determine the number of optimal genetic markers for PC calculation. 


\section{Conclusions}

In summary, we identified 17 risk loci for SCZ, including a Han Chinese-specific risk locus. We carried out comprehensive post-GWAS analysis, including TWAS, eQTL, differential expression analysis, and heritability partitioning (LDSC). Our results expand the list of genome-wide significant risk loci for SCZ and provide new insight into genetic architecture of SCZ.

\section{Abbreviations}

ASA: Asian Screening Array; CMC: CommonMind Consortium; EAS: East Asian ancestry; eQTL: Expression quantitative trait locus; EUR: European ancestry; GSA: Global Screening Array; GWAS: Genome-wide association studies; LDSC: Linkage disequilibrium score regression; PCA: Principal component analysis; PRS: Polygenic risk score; SCZ: Schizophrenia; TWAS: Transcriptomewide association study

\section{Supplementary Information}

The online version contains supplementary material available at https://doi. org/10.1186/s12916-021-02039-9.

Additional file 1: Figure S1. The flowchart of our quality control steps. Figure S2. The PCA results of the GSA group (831 cases and 1,700 controls). Figure S3. The PCA results of our samples (genotyped with ASA SNP array) and subjects from the 1000 Genome project (including CHB, CHS, JPT, CEU and YRI). Figure S4. The PCA results of our samples (genotyped with GSA SNP array) and subjects from the 1000 Genome project (including CHB, CHS, JPT, CEU and YRI). Figure S5. The QuantileQuantile plots of our Han Chinese samples. Figure S6. The allelic frequency of rs57016637 in global populations from the 1000 Genome project. Figure S7. The Manhattan plot of meta-analysis result of our Han Chinese samples and East Asian samples (26,271 cases and 40,071 controls). Figure S8. The locuszoom plot of rs3845188 ( $P=6.50 \times 10^{-8}, \mathrm{OR}=$ 0.91). Figure S9. Tissue and cell-type enrichment results. Table S1. The detail association result of the new genome wide significant loci identified in this study in different meta-analysis datasets. Table S2. Genes associated with the 17 newly identified lead SNPs in the human brain tissues. Table S3. Expression analysis of the potential eQTL target genes (of the newly identified lead SNPS) in schizophrenia cases and controls. Table S4. The MAGMA gene set enrichment analysis result (items with FDR $<0.10$ were listed). Table S5. The TWAS result. Significant genes (after Bonferroni correction) were listed.

\section{Acknowledgements}

One of the brain eQTL datasets used in this study was generated as part of the CommonMind Consortium supported by funding from Takeda Pharmaceuticals Company Limited, F. Hoffman-La Roche Ltd and NIH grants R01MH085542, R01MH093725, P50MH066392, P50MH080405, R01MH097276, RO1-MH-075916, P50M096891, P50MH084053S1, R37MH057881 and R37MH057881S1, HHSN271201300031C, AG02219, AG05138, and MH06692. Brain tissue for the study was obtained from the following brain bank collections: the Mount Sinai NIH Brain and Tissue Repository, the University of Pennsylvania Alzheimer's Disease Core Center, the University of Pittsburgh NeuroBioBank and Brain and Tissue Repositories and the NIMH Human Brain Collection Core. CMC Leadership: Pamela Sklar, Joseph Buxbaum (Icahn School of Medicine at Mount Sinai), Bernie Devlin, David Lewis (University of Pittsburgh), Raquel Gur, Chang-Gyu Hahn (University of Pennsylvania), Keisuke Hirai, Hiroyoshi Toyoshiba (Takeda Pharmaceuticals Company Limited), Enrico Domenici, Laurent Essioux (F. Hoffman-La Roche Ltd), Lara Mangravite, Mette Peters (Sage Bionetworks), Thomas Lehner, Barbara Lipska (NIMH). The Genotype-Tissue Expression (GTEx) Project was supported by the Common Fund of the Office of the Director of the National Institutes of Health, and by $\mathrm{NCl}, \mathrm{NHGRI}, \mathrm{NHLBI}, \mathrm{NIDA}, \mathrm{NIMH}$, and NINDS. We appreciate the Beijing Guoke Biotechnology Co, LTD (http://www.bioguoke.com/) for SNP genotyping. We are grateful for all the participants included in our study, and we thank Miss. Qian Li for her technical assistance.

\section{Authors' contributions}

X.J.L convinced, designed, and supervised the whole study. J.W.L performed most of the analyses, including QC, PCA, genome-wide association analysis, meta-analysis, LDSC, MAGMA analysis, and protein-protein interaction analysis. X.Y.L performed the TWAS and eQTL analyses. J.W.L drafted the manuscript, M.L, X.X, and Y.G.Y provided critical comments for manuscript improvement, X.J.L revised and finalized the manuscript. W.Q.L, Y.F.Y, S.Q.G, L.X.L, F.L.G, Y.G.Y, and M.L contributed partial schizophrenia cases to this work. All authors read this manuscript carefully, provided critical comments, and approved the manuscript.

\section{Funding}

This study was equally supported by the Distinguished Young Scientists grant of the Yunnan Province (202001AV070006) and the Key Research Project of Yunnan Province (202101AS070055). Also was supported by the Innovative Research Team of Science and Technology department of Yunnan Province (2019HC004), the Western Light Innovative Research Team of Chinses Academy of Sciences and the National Nature Science Foundation of China (31970561 to X.J.L, U1904130 to W.Q.L). We confirmed that all the funders were not involved in design of the study; and collection, analysis, and interpretation of data; and writing, submitting the manuscript.

\section{Availability of data and materials}

The datasets generated and/or analyzed during the current study are not publicly available due to the regulations of the Ministry of Science and Technology of the People's Republic of China (on human genetic resource usage). We need to comply with relevant regulations regarding the release of original genetic data (including the summary statistics) of the Chinese patients. This will take some time to finish according to the related policy. Data depositing in a third-party website (without application) is not allowed before approval. We understand that sharing of the genome-wide summary statistics publicly will contribute to the progress of the relevant field and we are planning to promote the progress of sharing summary statistics after this study is finalized. In addition, these GWAS samples were recruited from multiple hospitals across Mainland China, and this study could not have been completed without the great efforts from all the collaborators, we will need to get approval from all the authors (and their affiliated agencies) before sharing the summary statistics. However, these data are available from the corresponding author on reasonable request.

URLS:

PLINK, http://www.cog-genomics.org/plink2; KING, http://people.virginia. edu/ wC9c/KING/; EIGENSOFT, https://www.hsph.harvard.edu/alkes-price/ software/; GCTA, http://cnsgenomics.com/software/gcta/; The 1000 Genome project website, https://www.internationalgenome.org/; Minimac3, https:// genome.sph.umich.edu/wiki/Minimac3; LDSC, https://github.com/bulik/ldsc MAGMA, https://ctg.cncr.nl/software/magma; MSigDB, http://software. broadinstitute.org/gsea/msigdb/; PsychEncode, http://www.psychencode. org/:" Locuszoom, http://locuszoom.org; FUMA, https://fuma.ctglab.nl/. Single cell RNA-seq analysis, https://github.com/jbryois/scRNA_disease; PRSice2, http://www.prsice.info/; FUSION, http://gusevlab.org/projects/fusion/; SZDB, http://www.szdb.org

\section{Declarations}

Ethics approval and consent to participate

All participants provided written informed consents and this study was approved by the Ethical Committee and internal review board of the Kunming Institute of Zoology, the Second Affiliated Hospital of Xinxiang Medical University and Xi'an Jiaotong University. Ethics approval No: SMKX20191215-07.

\section{Consent for publication}

Not applicable.

\section{Competing interests}

The authors declare no competing interests and financial relationships with commercial interests.

\section{Author details}

${ }^{1}$ Key Laboratory of Animal Models and Human Disease Mechanisms of the Chinese Academy of Sciences \& Yunnan Province, Kunming Institute of 
Zoology, Chinese Academy of Sciences, Kunming 650223, Yunnan, China. ${ }^{2}$ Kunming College of Life Science, University of Chinese Academy of Sciences, Kunming 650204, Yunnan, China. ${ }^{3}$ Henan Mental Hospital, The Second Affiliated Hospital of Xinxiang Medical University, Xinxiang 453002, Henan, China. ${ }^{4}$ Henan Key Lab of Biological Psychiatry, International Joint Research Laboratory for Psychiatry and Neuroscience of Henan, Xinxiang Medical University, Xinxiang 453002, Henan, China. ${ }^{5}$ KIZ-CUHK Joint Laboratory of Bioresources and Molecular Research in Common Diseases, Kunming Institute of Zoology, Chinese Academy of Sciences, Kunming 650223, Yunnan, China. ${ }^{6}$ CAS Center for Excellence in Brain Science and Intelligence Technology, Chinese Academy of Sciences, Shanghai 200031, China. ${ }^{7}$ Department of Forensic Psychiatry, School of Medicine \& Forensics, Xi'an Jiaotong University Health Science Center, Xi'an 710061, Shaanxi, China ${ }^{8}$ Center for Excellence in Animal Evolution and Genetics, Chinese Academy of Sciences, Kunming 650223, China.

\section{Received: 16 March 2021 Accepted: 17 June 2021}

Published online: 12 August 2021

\section{References}

1. Saha S, Chant D, Welham J, McGrath J. A systematic review of the prevalence of schizophrenia. PLoS Med. 2005;2(5):e141. https://doi.org/10.13 71/journal.pmed.0020141.

2. Owen MJ, Sawa A, Mortensen PB. Schizophrenia. Lancet. 2016;388(10039): 86-97. https://doi.org/10.1016/S0140-6736(15)01121-6.

3. Laursen TM, Nordentoft M, Mortensen PB. Excess early mortality in schizophrenia. Annu Rev Clin Psychol. 2014;10(1):425-48. https://doi.org/1 0.1146/annurev-clinpsy-032813-153657.

4. Cloutier M, Aigbogun MS, Guerin A, Nitulescu R, Ramanakumar AV, Kamat SA, et al. The Economic Burden of Schizophrenia in the United States in 2013. J Clin Psychiatry. 2016;77(06):764-71. https://doi.org/10.4088/JCP.15m1 0278 .

5. Sullivan PF, Kendler KS, Neale MC. Schizophrenia as a complex trait: evidence from a meta-analysis of twin studies. Arch Gen Psychiatry. 2003; 60(12):1187-92. https://doi.org/10.1001/archpsyc.60.12.1187.

6. International Schizophrenia Consortium. Rare chromosomal deletions and duplications increase risk of schizophrenia. Nature. 2008;455(7210):237-41. https://doi.org/10.1038/nature07239.

7. McCarthy SE, Makarov V, Kirov G, Addington AM, McClellan J, Yoon S, et al. Microduplications of $16 p 11.2$ are associated with schizophrenia. Nat Genet. 2009;41:1223-7.

8. Stefansson H, Rujescu D, Cichon S, Pietiläinen OP, Ingason A, Steinberg S, et al. Large recurrent microdeletions associated with schizophrenia. Nature. 2008;455(7210):232-6. https://doi.org/10.1038/nature07229.

9. Walsh T, McClellan JM, McCarthy SE, Addington AM, Pierce SB, Cooper GM, et al. Rare structural variants disrupt multiple genes in neurodevelopmental pathways in schizophrenia. Science. 2008;320(5875):539-43. https://doi.org/1 $0.1126 /$ science. 1155174.

10. Xu B, Roos JL, Levy S, van Rensburg EJ, Gogos JA, Karayiorgou M. Strong association of de novo copy number mutations with sporadic schizophrenia. Nat Genet. 2008;40(7):880-5. https://doi.org/10.1038/ng.162.

11. Purcell SM, Moran JL, Fromer M, Ruderfer D, Solovieff N, Roussos P, et al. A polygenic burden of rare disruptive mutations in schizophrenia. Nature. 2014;506(7487):185-90. https://doi.org/10.1038/nature12975.

12. Xu B, lonita-Laza I, Roos JL, Boone B, Woodrick S, Sun Y, et al. De novo gene mutations highlight patterns of genetic and neural complexity in schizophrenia. Nat Genet. 2012;44(12):1365-9. https://doi.org/10.1038/ng.244 6.

13. Gulsuner S, Walsh T, Watts AC, Lee MK, Thornton AM, Casadei S, et al. Spatial and temporal mapping of de novo mutations in schizophrenia to a fetal prefrontal cortical network. Cell. 2013;154(3):518-29. https://doi.org/10.1 016/j.cell.2013.06.049.

14. Fromer M, Pocklington AJ, Kavanagh DH, Williams HJ, Dwyer S, Gormley P, et al. De novo mutations in schizophrenia implicate synaptic networks. Nature. 2014;506(7487):179-84. https://doi.org/10.1038/nature12929.

15. Gulsuner S, Stein DJ, Susser ES, Sibeko G, Pretorius A, Walsh T, et al. Genetics of schizophrenia in the South African Xhosa. Science. 2020;367(6477):56973. https://doi.org/10.1126/science.aay8833.

16. Singh T, Kurki Ml, Curtis D, Purcell SM, Crooks L, McRae J, et al. Rare loss-offunction variants in SETD1A are associated with schizophrenia and developmental disorders. Nat Neurosci. 2016;19(4):571-7. https://doi.org/1 $0.1038 / \mathrm{nn} .4267$

17. Yu H, Yan H, Li J, Li Z, Zhang X, Ma Y, et al. Common variants on 2p16.1, 6p22.1 and 10q24.32 are associated with schizophrenia in Han Chinese population. Mol Psychiatry. 2017;22(7):954-60. https://doi.org/10.1038/mp.2 016.212.

18. Stefansson H, Ophoff RA, Steinberg S, Andreassen OA, Cichon S, Rujescu D, et al. Common variants conferring risk of schizophrenia. Nature. 2009; 460(7256):744-7. https://doi.org/10.1038/nature08186.

19. Shi Y, Li Z, Xu Q, Wang T, Li T, Shen J, et al. Common variants on 8p12 and 1q24.2 confer risk of schizophrenia. Nat Genet. 2011;43(12):1224-7. https:// doi.org/10.1038/ng.980.

20. International Schizophrenia Consortium, Purcell SM, Wray NR, Stone JL, Visscher PM, O'Donovan MC, et al. Common polygenic variation contributes to risk of schizophrenia and bipolar disorder. Nature. 2009:460:748-52.

21. Pardiñas AF, Holmans P, Pocklington AJ, Escott-Price V, Ripke S, Carrera N, et al. Common schizophrenia alleles are enriched in mutation-intolerant genes and in regions under strong background selection. Nat Genet. 2018; 50(3):381-9. https://doi.org/10.1038/s41588-018-0059-2.

22. O'Donovan MC, Craddock N, Norton N, Williams H, Peirce T, Moskvina V, et al. Identification of loci associated with schizophrenia by genome-wide association and follow-up. Nat Genet. 2008;40(9):1053-5. https://doi.org/10.1 038/ng.201.

23. Li Z, Chen J, Yu H, He L, Xu Y, Zhang D, et al. Genome-wide association analysis identifies 30 new susceptibility loci for schizophrenia. Nat Genet. 2017;49(11):1576-83. https://doi.org/10.1038/ng.3973.

24. Lam M, Chen CY, Li Z, Martin AR, Bryois J, Ma X, et al. Comparative genetic architectures of schizophrenia in East Asian and European populations. Nat Genet. 2019;51(12):1670-8. https://doi.org/10.1038/s41588-019-0512-X.

25. Hamshere ML, Walters JT, Smith R, Richards AL, Green E, Grozeva D, et al. Genome-wide significant associations in schizophrenia to ITIH3/4, CACNA1C and SDCCAG8, and extensive replication of associations reported by the Schizophrenia PGC. Mol Psychiatry. 2013;18(6):708-12. https://doi.org/10.103 8/mp.2012.67.

26. Schizophrenia Working Group of the Psychiatric Genomics Consortium. Biological insights from 108 schizophrenia-associated genetic loci. Nature. 2014;511(7510):421-7. https://doi.org/10.1038/nature13595.

27. Schizophrenia Psychiatric Genome-Wide Association Study Consortium. Genome-wide association study identifies five new schizophrenia loci. Nat Genet. 2011:43(10):969-76. https://doi.org/10.1038/ng.940.

28. Genomes Project Consortium, Auton A, Brooks LD, Durbin RM, Garrison EP, Kang HM, et al. A global reference for human genetic variation. Nature. 2015;526:68-74

29. Huckins LM, Dobbyn A, Ruderfer DM, Hoffman G, Wang W, Pardiñas AF, et al. Gene expression imputation across multiple brain regions provides insights into schizophrenia risk. Nat Genet. 2019;51(4):659-74. https://doi. org/10.1038/s41588-019-0364-4.

30. Luo XJ, Mattheisen M, Li M, Huang L, Rietschel M, Børglum AD, et al. Systematic integration of brain eQTL and GWAS identifies ZNF323 as a novel schizophrenia risk gene and suggests recent positive selection based on compensatory advantage on pulmonary function. Schizophr Bull. 2015; 41(6):1294-308. https://doi.org/10.1093/schbul/sbv017.

31. Yang CP, Li X, Wu Y, Shen Q, Zeng Y, Xiong Q, et al. Comprehensive integrative analyses identify GLT8D1 and CSNK2B as schizophrenia risk genes. Nat Commun. 2018;9(1):838. https://doi.org/10.1038/s41467-018-0324 7-3.

32. Li M, Luo XJ, Xiao X, Shi L, Liu XY, Yin LD, et al. Allelic differences between Han Chinese and Europeans for functional variants in ZNF804A and their association with schizophrenia. Am J Psychiatry. 2011;168(12):1318-25. https://doi.org/10.1176/appi.ajp.2011.11030381

33. Luo XJ, Diao HB, Wang JK, Zhang H, Zhao ZM, Su B. Association of haplotypes spanning PDZ-GEF2, LOC728637 and ACSL6 with schizophrenia in Han Chinese. J Med Genet. 2008;45(12):818-26. https://doi.org/10.1136/ jmg.2008.060657.

34. Li K, Li Y, Wang J, Huo Y, Huang D, Li S, et al. A functional missense variant in ITIH3 affects protein expression and neurodevelopment and confers schizophrenia risk in the Han Chinese population. J Genet Genomics. 2020; 47(5):233-48. https://doi.org/10.1016/j.jgg.2020.04.001.

35. Anderson CA, Pettersson FH, Clarke GM, Cardon LR, Morris AP, Zondervan KT. Data quality control in genetic case-control association studies. Nat Protoc. 2010;5(9):1564-73. https://doi.org/10.1038/nprot.2010.116. 
36. Manichaikul A, Mychaleckyj JC, Rich SS, Daly K, Sale M, Chen WM. Robust relationship inference in genome-wide association studies. Bioinformatics. 2010;26(22):2867-73. https://doi.org/10.1093/bioinformatics/btq559.

37. Purcell S, Neale B, Todd-Brown K, Thomas L, Ferreira MA, Bender D, et al. PLINK: a tool set for whole-genome association and population-based linkage analyses. Am J Hum Genet. 2007;81(3):559-75. https://doi.org/10.1 086/519795.

38. Auton A, Brooks LD, Durbin RM, Garrison EP, Kang HM, Korbel JO, et al. A global reference for human genetic variation. Nature. 2015;526(7571):68-74. https://doi.org/10.1038/nature15393.

39. Yang J, Lee SH, Goddard ME, Visscher PM. GCTA: a tool for genome-wide complex trait analysis. Am J Hum Genet. 2011;88(1):76-82. https://doi.org/1 0.1016/j.ajhg.2010.11.011.

40. Patterson N, Price AL, Reich D. Population structure and eigenanalysis. PLoS Genet. 2006;2(12):e190. https://doi.org/10.1371/journal.pgen.0020190.

41. Price AL, Patterson NJ, Plenge RM, Weinblatt ME, Shadick NA, Reich D. Principal components analysis corrects for stratification in genome-wide association studies. Nat Genet. 2006;38(8):904-9. https://doi.org/10.1038/ng1 847.

42. Price AL, Zaitlen NA, Reich D, Patterson N. New approaches to population stratification in genome-wide association studies. Nat Rev Genet. 2010;11(7): 459-63. https://doi.org/10.1038/nrg2813.

43. Kang HM, Sul JH, Service SK, Zaitlen NA, Kong SY, Freimer NB, et al. Variance component model to account for sample structure in genome-wide association studies. Nat Genet. 2010;42(4):348-54. https://doi.org/10.1038/ ng.548.

44. Loh PR, Danecek P, Palamara PF, Fuchsberger C, Reshef YA, Finucane HK, et al. Reference-based phasing using the Haplotype Reference Consortium panel. Nat Genet. 2016;48(11):1443-8. https://doi.org/10.1038/ng.3679.

45. Das S, Forer L, Schönherr S, Sidore C, Locke AE, Kwong A, et al. Nextgeneration genotype imputation service and methods. Nat Genet. 2016; 48(10):1284-7. https://doi.org/10.1038/ng.3656.

46. Watanabe K, Taskesen E, van Bochoven A, Posthuma D. Functional mapping and annotation of genetic associations with FUMA. Nat Commun. 2017;8(1): 1826. https://doi.org/10.1038/s41467-017-01261-5.

47. Pruim RJ, Welch RP, Sanna S, Teslovich TM, Chines PS, Gliedt TP, et al. LocusZoom: regional visualization of genome-wide association scan results. Bioinformatics. 2010;26(18):2336-7. https://doi.org/10.1093/bioinformatics/ btq419.

48. Choi SW, O'Reilly PF. PRSice-2: Polygenic Risk Score software for biobankscale data. Gigascience. 2019;8(7). https://doi.org/10.1093/gigascience/ giz082.

49. de Leeuw CA, Mooij JM, Heskes T, Posthuma D. MAGMA: generalized geneset analysis of GWAS data. PLoS Comput Biol. 2015:11(4):e1004219. https:// doi.org/10.1371/journal.pcbi.1004219.

50. GTEx Consortium. The Genotype-Tissue Expression (GTEx) project. Nat Genet. 2013;45:580-5.

51. Zeisel A, Hochgerner H, Lönnerberg P, Johnsson A, Memic F, van der Zwan J, et al. Molecular Architecture of the Mouse Nervous System. Cell. 2018;174: 999-1014.e22

52. Bryois J, Skene NG, Hansen TF, Kogelman LJA, Watson HJ, Liu Z, et al. Genetic identification of cell types underlying brain complex traits yields insights into the etiology of Parkinson's disease. Nat Genet. 2020;52(5):48293. https://doi.org/10.1038/s41588-020-0610-9.

53. Subramanian A, Tamayo P, Mootha VK, Mukherjee S, Ebert BL, Gillette MA, et al. Gene set enrichment analysis: a knowledge-based approach for interpreting genome-wide expression profiles. Proc Natl Acad Sci U S A. 2005;102(43):15545-50. https://doi.org/10.1073/pnas.0506580102.

54. Finucane HK, Bulik-Sullivan B, Gusev A, Trynka G, Reshef $Y$, Loh PR, et al. Partitioning heritability by functional annotation using genome-wide association summary statistics. Nat Genet. 2015;47(11):1228-35. https://doi. org/10.1038/ng.3404

55. Fromer M, Roussos P, Sieberts SK, Johnson JS, Kavanagh DH, Perumal TM, et al. Gene expression elucidates functional impact of polygenic risk for schizophrenia. Nat Neurosci. 2016;19(11):1442-53. https://doi.org/10.1038/ nn.4399.

56. Gusev A, Ko A, Shi H, Bhatia G, Chung W, Penninx BW, et al. Integrative approaches for large-scale transcriptome-wide association studies. Nat Genet. 2016;48(3):245-52. https://doi.org/10.1038/ng.3506.

57. Collado-Torres L, Burke EE, Peterson A, Shin J, Straub RE, Rajpurohit A, et al. Regional heterogeneity in gene expression, regulation, and coherence in the frontal cortex and hippocampus across development and schizophrenia. Neuron. 2019;103:203-16.e8.

58. Ng B, White CC, Klein HU, Sieberts SK, McCabe C, Patrick E, et al. An xQTL map integrates the genetic architecture of the human brain's transcriptome and epigenome. Nat Neurosci. 2017;20(10):1418-26. https://doi.org/10.1038/ nn.4632.

59. Wang D, Liu S, Warrell J, Won H, Shi X, Navarro FCP, et al. Comprehensive functional genomic resource and integrative model for the human brain. Science. 2018;362(6420):eaat8464. https://doi.org/10.1126/science.aat8464.

60. Yue WH, Wang HF, Sun LD, Tang FL, Liu ZH, Zhang HX, et al. Genome-wide association study identifies a susceptibility locus for schizophrenia in Han Chinese at 11p11.2. Nat Genet. 2011;43(12):1228-31. https://doi.org/10.1038/ ng.979.

61. von Engelhardt J, Mack V, Sprengel R, Kavenstock N, Li KW, Stern-Bach Y, et al. CKAMP44: a brain-specific protein attenuating short-term synaptic plasticity in the dentate gyrus. Science. 2010;327(5972):1518-22. https://doi. org/10.1126/science.1184178.

62. Hammond JC, McCullumsmith RE, Funk AJ, Haroutunian V, MeadorWoodruff $\mathrm{JH}$. Evidence for abnormal forward trafficking of AMPA receptors in frontal cortex of elderly patients with schizophrenia. Neuropsychopharmacology. 2010;35(10):2110-9. https://doi.org/10.1038/ npp.2010.87.

63. Mhalla A, Bel Hadj Salah W, Mensi R, Amamou B, Messaoud A, Gassab L, et al. Lipid profile in schizophrenia: case control study. Tunis Med. 2018; 96(1):22-9.

64. Krakowski M, Czobor P. Cholesterol and cognition in schizophrenia: a double-blind study of patients randomized to clozapine, olanzapine and haloperidol. Schizophr Res. 2011;130(1-3):27-33. https://doi.org/10.1016/j. schres.2011.04.005

65. Ripke S, Walters JT, O'Donovan MC. Mapping genomic loci prioritises genes and implicates synaptic biology in schizophrenia. medRxiv. 2020:2020.09.12. 20192922. https://doi.org/10.1101/2020.09.12.20192922

66. Andreasen NC, Pierson R. The role of the cerebellum in schizophrenia. Bio Psychiatry. 2008;64(2):81-8. https://doi.org/10.1016/j.biopsych.2008.01.003.

67. Kim DJ, Kent JS, Bolbecker AR, Sporns O, Cheng H, Newman SD, et al. Disrupted modular architecture of cerebellum in schizophrenia: a graph theoretic analysis. Schizophr Bull. 2014;40(6):1216-26. https://doi.org/10.1 093/schbul/sbu059.

68. Martin P, Albers M. Cerebellum and schizophrenia: a selective review. Schizophr Bull. 1995;21(2):241-50. https://doi.org/10.1093/schbul/21.2.241.

69. Picard H, Amado I, Mouchet-Mages S, Olié JP, Krebs MO. The role of the cerebellum in schizophrenia: an update of clinical, cognitive, and functional evidences. Schizophr Bull. 2008;34(1):155-72. https://doi.org/10.1093/schbul/ sbm049.

70. Zhao H, Mitra N, Kanetsky PA, Nathanson KL, Rebbeck TR. A practical approach to adjusting for population stratification in genome-wide association studies: principal components and propensity scores (PCAPS). Stat Appl Genet Mol Biol. 2018;17(6):20170054. https://doi.org/10.1515/sa gmb-2017-0054

\section{Publisher's Note}

Springer Nature remains neutral with regard to jurisdictional claims in published maps and institutional affiliations.

Ready to submit your research? Choose BMC and benefit from:

- fast, convenient online submission

- thorough peer review by experienced researchers in your field

- rapid publication on acceptance

- support for research data, including large and complex data types

- gold Open Access which fosters wider collaboration and increased citations

- maximum visibility for your research: over $100 \mathrm{M}$ website views per year

At $\mathrm{BMC}$, research is always in progress.

Learn more biomedcentral.com/submission 